\title{
Mobile Colistin Resistance Genetic Determinants of Non-Typhoid Salmonella enterica Isolates from Russia
}

\author{
Konstantin V. Kuleshov ${ }^{1,2, *(\mathbb{D}}$, Anastasia S. Pavlova ${ }^{1}\left(\mathbb{D}\right.$, Elizaveta D. Shedko ${ }^{1}{ }^{(D}$, Yulia V. Mikhaylova ${ }^{1}{ }^{(}$, \\ Gabriele Margos ${ }^{3}$, Sabrina Hepner ${ }^{3}$, Igor V. Chebotar ${ }^{4}{ }^{(}$, Elena V. Korneenko ${ }^{1}$, Alexander T. Podkolzin ${ }^{1}$ \\ and Vasiliy G. Akimkin ${ }^{1}$ \\ 1 Central Research Institute of Epidemiology, 111123 Moscow, Russia; nasty-pavlov@yandex.ru (A.S.P.); \\ hardcandy.hd@gmail.com (E.D.S.); yulka_ivashka@mail.ru (Y.V.M.); lennatta@yandex.ru (E.V.K.); \\ podkolzin@pcr.ru (A.T.P.); vgakimkin@yandex.ru (V.G.A.) \\ 2 Federal State Budget Scientific Institution "Federal Scientific Center VIEV", 109428 Moscow, Russia \\ 3 German National Reference Centre for Borrelia, Bavarian Health and Food Safety Authority, Veterinärstr. 2, \\ 85764 Oberschleissheim, Germany; gmargos1@gmail.com (G.M.); sabrina.hepner@lgl.bayern.de (S.H.) \\ 4 Laboratory of Molecular Microbiology, Pirogov Russian National Research Medical University, \\ 117997 Moscow, Russia; nizarnn@yandex.ru \\ * Correspondence: konstantinkul@gmail.com
}

Citation: Kuleshov, K.V.; Pavlova, A.S.; Shedko, E.D.; Mikhaylova, Y.V.; Margos, G.; Hepner, S.; Chebotar, I.V.; Korneenko, E.V.; Podkolzin, A.T.; Akimkin, V.G. Mobile Colistin Resistance Genetic Determinants of Non-Typhoid Salmonella enterica Isolates from Russia. Microorganisms 2021, 9, 2515. https://doi.org/ 10.3390/ microorganisms 9122515

Academic Editor: Gabriela Jorge Da Silva

Received: 7 November 2021

Accepted: 1 December 2021

Published: 4 December 2021

Publisher's Note: MDPI stays neutral with regard to jurisdictional claims in published maps and institutional affiliations.

Copyright: (c) 2021 by the authors. Licensee MDPI, Basel, Switzerland. This article is an open access article distributed under the terms and conditions of the Creative Commons Attribution (CC BY) license (https:// creativecommons.org/licenses/by/ $4.0 /)$.
Abstract: Polymyxin resistance, determined by $\mathrm{mcr}$ genes located on plasmid DNA, currently poses a high epidemiological threat. Non-typhoid Salmonella (NTS) are one of the key pathogens causing diarrheal diseases. Here, we report the isolation and whole genome sequencing of multidrug colistinresistant/susceptible isolates of non-typhoid Salmonella enterica serovars carrying mor genes. Nontyphoid strains of Salmonella enterica subsp. enterica were isolated during microbiological monitoring of the environment, food, and diarrheal disease patients between 2018 and 2020 in Russia $(n=586)$. $m c r-1$ genes were detected using a previously developed qPCR assay, and whole genome sequencing of $\mathrm{mcr}$ positive isolates was performed by both short-read (Illumina) and long-read (Oxford Nanopore) approaches. Three colistin-resistant isolates, including two isolates of $S$. Enteritidis and one isolate of $S$. Bovismorbificans, carried the $m c r-1.1$ gene located on IncX4 and IncI2 conjugative plasmids, respectively. The phenotypically colistin-susceptible isolate of $S$. Typhimurium carried a $m c r-9$ gene on plasmid IncHI2. In conclusion, we present the first three cases of $\mathrm{mcr}$ gene-carrying NTS isolates detected in Russia with both outbreak and sporadic epidemiological backgrounds.

Keywords: whole genome sequencing; antibiotic resistance; Salmonella Enteritidis; Salmonella Typhimurium; Salmonella Bovismorbificans; colistin resistance; $m c r-1 ;$ mcr-9

\section{Introduction}

Non-typhoid strains of Salmonella enterica (NTS) contribute significantly to the incidence of intestinal infections worldwide [1]. Globally, there are more than 90 million cases of Salmonella gastroenteritis each year, resulting in 155,000 deaths [2]. Despite a trend towards a decrease in salmonellosis, it is still the most frequently recorded foodborne zoonosis in Russia. The annual incidence rate of salmonellosis in Russia decreased from 36.6 per 100,000 population in 2012 to 22.0 per 100,000 in 2017 , but gradually increased in 2018 and 2019 up to 24.2 per 100,000. In 2020 and 2021, the annual incidence rate of salmonellosis decreased to 14.7 per 100,000 population [3]. During that time period, the prevailing serotypes in the etiological structure of morbidity remained Salmonella enterica subsp. enterica serotype Enteritidis and serotype Typhimurium [4].

The epidemiological and clinical relevance of NTS is determined by the genomic plasticity that underlies the adaptation to a wide host range, and the emergence of Salmonella strains with novel resistant profiles, in particular, the emergence of strains with plasmidmediated colistin resistance [5-7]. Importantly, in clinical practice, colistin is not used for 
the treatment of salmonellosis but it is a last resort drug in the treatment of infections associated with antibiotic-resistant Gram-negative bacteria and classified by the World Health Organization as "critically important." [8] Thus, Salmonella strains with plasmid encoding genes that confer resistance to colistin might be a potentially perilous reservoir for resistance determinants that can be moved via horizontal gene transfer among Gram-negative bacteria.

The first report on a plasmid-located mcr-1 gene in colistin-resistant Escherichia coli, obtained from livestock and patients in China, was made by Lui et al. in 2015 [9]. Subsequently, worldwide dissemination of $m c r-1$ positive strains in Enterobacteriaceae was shown [10]. To date, NTS strains of different serovars harboring mcr variants have been isolated from the environment, animals, and humans in various countries, indicating that such NTS may spread mor genes into diverse environmental niches worldwide [7].

Most $m c r$-carrying plasmids belong to the incompatibility plasmid group Inc [11]. A main feature of plasmids belonging to the Inc group is the presence of several toxinantitoxin systems which enable a high level of plasmid gene expression, and have resistance genes often located under the same promoter [12,13]. Among the studied plasmids with mor genes, the most common are IncX4 (35.2\%), IncI2 (34.7\%), and IncHI2 (20.5\%), with the IncI2 group prevailing in Asia (65.8\%) and IncHI2 in Europe (73.3\%) [14]. It is important to highlight that Inc plasmids were shown to carry both $\mathrm{mcr}$ and $\beta$-lactamase genes simultaneously $[15,16]$. In $77.8 \%$ of the studied IncHI2 plasmids and $37.9 \%$ of the IncI 2 ones, the insertion sequence ISApl1 was detected near the $m c r$ gene, yet it was always absent in IncX4 plasmids [14]. The most common elements that are found in the mcr cassette are the PIN-forming sequence $h p$, pilli folding protein IV type pilP, and the secretion system proteins IV type virD4 and virB4 [6,17].

Data from Russia of NTS strains circulating in the environment that carry genetic determinants of colistin resistance have so far been lacking. The aim of our study was to characterize the first four cases of $m c r$ gene-carrying NTS isolates detected in Russia with both outbreak and sporadic epidemiological background.

\section{Materials and Methods}

\subsection{Bacterial Isolation and Identification}

The collection included Salmonella strains collected by the All-Russia Reference Center of Salmonellosis during the national Salmonellosis surveillance program. In general, the collection of isolates $(n=586)$ included strains derived from humans $(n=293,58.6 \%)$, food ( $n=208,35.4 \%)$, environmental samples $(n=81,13.8 \%)$, and animals $(n=3,0.5 \%)$. The Salmonella isolated from humans included 139 representative isolates from 59 salmonellosis outbreaks (approximately two isolates from each outbreak) and 154 isolates from sporadic cases. S. Enteritidis was the most prevalent serotype among the outbreak strains (74.8\%).

All isolates were screened using McConkey medium. Individual colonies were used for re-identification and serotyping. Subsequently, each isolate was subjected to serological characterization according to the Kauffmann-White scheme using polyclonal (Petsal, St. Petersburg, Russia) and monoclonal antibodies (Sifin, Berlin, Germany).

\subsection{Antibiotic Susceptibility Testing}

Antibiotic susceptibility was tested by determining the minimum inhibitory concentration (MIC) for antibiotic susceptibility using GI and GII Mikrolatest ${ }^{\circledR}$ SensiLaTest MIC panels (Erba Lachema, Brno, Czech Republic). Escherichia coli (ATCC 25922) was used as an internal quality control. Isolates were tested on 19 antibiotics grouped into ten classes. Ssusceptibility interpretation criteria were used in accordance with the European Committee on Antimicrobial Susceptibility (EUCAST) interpretative criteria [18]. The double-disk synergy test (DDST) was employed to confirm the extended-spectrum beta-lactamase (ESBL) production using antimicrobial disks of ceftazidime $(30 \mu \mathrm{g})$, cefepime $(30 \mu \mathrm{g})$, and amoxicillin-clavulanic acid $(20 / 10 \mu \mathrm{g})$. Expansion of the indicator cephalosporin inhibi- 
tion zone towards the amoxicillin-clavulanic acid disk was considered indicative of ESBL production [19].

\subsection{Real-Time PCR Assays for mor-1}

Salmonella isolates were tested for the presence of $m c r-1$ group genes. DNA extraction and real-time polymerase chain reaction (qPCR) was performed using the PCR-kit «AmpliSens ${ }^{\circledR}$ MDR MCR-1-FL» (Central Research Institute of Epidemiology, Moscow, Russia). The qPCR assay was performed on a Rotor-Gene ${ }^{\circledR} 6000$ (Corbett Research, Mortlake, Australia). Data obtained with all used reagent kits was analyzed using the RotorGene 6000 v1.8 software, according to the manufacturer's instructions.

\subsection{Whole-Genome Sequencing and Assembling}

Salmonella isolates were subjected to HiSeq (Illumina, San Francisco, CA, USA) and MinION (Oxford Nanopore, Oxford, UK) sequencing technology. Genomic DNA purification was performed using a DNeasy ${ }^{\circledR}$ Blood \& Tissue Kit (Qiagen, Hilden, Germany), according to the manufacturer's instructions. The purified genomic DNA was used for both whole genome sequencing technologies (Illumina and Oxford Nanopore). The libraries for Illumina sequencing were prepared using the Nextera XT DNA Library Prep Kit (Illumina, USA), Index Kit (Illumina, USA), and Reagent Kit (Illumina, USA). The sequencing was performed using a HiSeq 2500 instrument that produced $2 \times 250$ base pair (bp) paired-end reads.

The DNA library for MinION sequencing was prepared using the Native Barcoding Kit 1D (EXP NBD104, Oxford Nanopore) and Ligation Sequencing Kit 1D (SQK-LSK109, Oxford Nanopore, Oxford, UK), according to the manufacturer's protocol, then sequenced using an R9 SpotON flow cell (FLO-MIN106). The raw FAST5 reads were basecalled using the Guppy Basecalling Software v3.4.4. The reads were then subsequently trimmed by Porechop (https:/ / github.com/rrwick/Porechop) (accessed on 1 November 2020) with the flag 'discard_middle,' and filtered on the quality and read length by Nanofilt. The quality threshold was defined as the median quality of initial sets of raw long reads of a particular isolate, while the minimum read length was set to $1000 \mathrm{bp}$. Accession numbers for the short-read and long-read data for each isolate are available under the following accession numbers: SLR1_8250 - SRR12342988 (long-reads), SRR12342993 (short-reads), SLR1_8245 - SRR15212457, SRR15212459, SLR1_7627 - SRR12342987, SRR12342992, SLR1_8094 SRR12342986, SRR12342991.

\subsection{Bioinformatic Analysis}

Unicycler v0.4.2 [20] was used to generate a hybrid assembly, combining the long reads (Oxford Nanopore) and short reads (Illumina). Independently, Canu v1.8 [21] assemblies using only the Oxford Nanopore long reads, and subsequent dot-plot analyses using Flexidot [22], were performed to compare assembly results (Unicycler versus Canu). The comparison parameter was the topology and approximate length of the assembled chromosomes and plasmids.

The sequenced genomes were submitted to GenBank and annotated by the prokaryotic annotation pipeline (PGAP). These genomes were automatically imported to the NCBI Pathogen Detection system (https://www.ncbi.nlm.nih.gov/pathogens/) (accessed on 29 November 2021) and to EnteroBase (https: / / enterobase.warwick.ac.uk/species/index/ senterica) (accessed on 29 November 2021). Using EnteroBase's information, we could identify the sequence types (ST) defined by Achtman's seven gene MLST scheme, as well as the eBURST groups (eBG) for each strain [23]. These systems also provided results by searching for clonally-related isolates via incorporated bioinformatic pipelines. The NCBI Pathogen Detection system used an SNP-based approach, while EnteroBase used a core genome MLST (cgMLST). It should be noted that in the SNP-based and core genome MLST comparative analysis we also included an isolate of S. Enteritidis SLR1_8239 (accession 
numbers for the short-read SRR15212458), which originated from the same outbreak as SLR1_8245, to demonstrate clonal relationships between isolates.

Each assembled genome was submitted for analyses using the MOB-suite v3.0.0 [24] and PlasmidFinder v1.3 [25] to define replicon types, and to predict mobility for the assembled plasmids.

Screening for antimicrobial resistance (AMR) genes and point mutations were performed by several approaches using ResFinder v4.0 [26] and AMRFinderPlus v3.6.10 [27].

To determine whether differences may exist between the plasmids harboring $\mathrm{mcr}$ genes, annotated sequences of plasmids were visualized and compared in the Easyfig software v.2.1 [28] or BLAST Ring Image Generator (BRIG) [29].

\section{Results}

\subsection{Isolates Description and Antimicrobial Susceptibility Testing}

During microbiological monitoring of 85 federal subjects of the Russian Federation between 2018 and 2020 by the All-Russian Salmonella Reference Laboratory, NTS isolates $(n=586)$ were collected from the environment, food, and patients. All isolates were subjected to antibiotic susceptibility testing, as well as screened for the presence of $\mathrm{mcr}$ 1-type by a specific qPCR. In this screen, only three isolates ( $S$. Enteritidis $(n=2), S$. Bovismorbificans $(n=1)$ ) were shown to carry an $m c r-1$ gene (Table 1 ). In our study, we also included an isolate, SLR1_8094, representing a S. Typhimurium with an $m c r-9$ gene, which was isolated from a sporadic case in 2019. The presence of an $m c r-9$ gene in its genome was discovered during a whole genome sequencing study of Salmonella isolates circulating in Russia, which were characterized as a multidrug-resistant phenotype.

Table 1. Russian isolates characterized by presence of $m c r$ genes.

\begin{tabular}{|c|c|c|c|c|c|c|}
\hline & Serotype & $\begin{array}{c}\text { Isolation Date } \\
\text { (mm/yyyy) }\end{array}$ & Country:City & $\begin{array}{l}\text { Epidemiological } \\
\text { Background }\end{array}$ & Source & mcr-Type \\
\hline SLR1_8250 & S. Enteritidis & $04 / 2019$ & Russia:Yakutiya & ND & $\begin{array}{l}\text { food (chicken } \\
\text { meat) }\end{array}$ & 1 \\
\hline SLR1_8245 & S. Enteritidis & $07 / 2019$ & Russia:El'ban & Outbreak & human & 1 \\
\hline SLR1_7627 & S. Bovismorbificans & $09 / 2018$ & Russia:Irkutsk & Sporadic & human & 1 \\
\hline SLR1_8094 & $\begin{array}{c}\text { S. Typhimurium } \\
\text { monophasic variant } \\
{[4,5: \mathrm{i}:-]}\end{array}$ & 06/2019 & Russia:Ulan-Ude & Sporadic & human & 9 \\
\hline
\end{tabular}

$$
\text { ND—data not available. }
$$

While the $m c r$-1 positive S. Enteritidis SLR1_8250 and S. Bovismorbificans SLR1_7627 strains were isolated from sporadic cases, it should be noted that the $\mathrm{mcr}-1$ positive strain SLR1_8245 (S. Enteritidis) was a representative isolate from an outbreak of salmonellosis with 239 affected people in El'ban village in the Khabarovsk Territory.

Isolates carrying the $m c r-1$ gene are characterized by high MIC values to colistin (MIC $8 \mathrm{mg} / \mathrm{L}$ ) in antimicrobial susceptibility testing (AST). On the contrary, the isolate with the $m c r-9$ gene showed no resistance to colistin (MIC $1 \mathrm{mg} / \mathrm{L}$ ) (Table 2). SLR1_8094 produced ESBL which was confirmed by DDST. Moreover, according to our data, only isolate SLR1_8250 was resistant to a single drug-namely colistin-while the other three isolates showed a multidrug-resistant (MDR) phenotype defined as resistance to drugs of at least three different antimicrobial classes. An extended spectrum of resistance to 11 antimicrobial agents, representing seven antimicrobial classes, was detected in the monophasic variant S. Typhimurium SLR1_8094 (Table 2). 
Table 2. Antimicrobial susceptibility testing of four Salmonella isolates containing an mor gene based on MIC and AMR genes associated with resistance to particular antimicrobial agents.

\begin{tabular}{|c|c|c|c|c|c|c|c|c|c|}
\hline \multirow{2}{*}{$\begin{array}{c}\begin{array}{c}\text { Antimicrobial } \\
\text { Class }\end{array} \\
\text { polymyxins }\end{array}$} & \multirow{2}{*}{$\begin{array}{c}\text { Antimicrobial Agent } \\
\text { colistin }\end{array}$} & \multicolumn{2}{|c|}{ SLR1_8250 } & \multicolumn{2}{|c|}{ SLR1_7627 } & \multicolumn{2}{|c|}{ SLR1_8245 } & \multicolumn{2}{|c|}{ SLR1_8094 } \\
\hline & & $8(\mathrm{R})$ & mcr-1.1 & $8(\mathrm{R})$ & mcr-1.1 & $8(\mathrm{R})$ & mcr-1.1 & $1(\mathrm{~S})$ & mcr-9 \\
\hline \multirow{4}{*}{ penicillins } & ampicillin & $2(S)$ & & $>128(\mathrm{R})$ & \multirow{4}{*}{ blaTEM-1B } & $2(S)$ & & >128 (R) & \multirow{4}{*}{$\begin{array}{l}\text { blaDHA-1, } \\
\text { blaSHV-12 }\end{array}$} \\
\hline & ampicillin-sulbactam & $2 / 1(\mathrm{~S})$ & & $32 / 8(\mathrm{R})$ & & $2 / 1(S)$ & & $64 / 32(\mathrm{R})$ & \\
\hline & piperacillin & $2(S)$ & & $>128(\mathrm{R})$ & & $2(\mathrm{~S})$ & & $>128(\mathrm{R})$ & \\
\hline & piperacillin-tazobactam & $\leq 2 / 4(\mathrm{~S})$ & & $\leq 1 / 4(\mathrm{~S})$ & & $2 / 4(S)$ & & $2 / 4(\mathrm{~S})$ & \\
\hline \multirow{3}{*}{ cephems } & cefotaxime & $0.12(\mathrm{~S})$ & & $0.12(\mathrm{~S})$ & & $0.12(\mathrm{~S})$ & & $>8(\mathrm{R})$ & \multirow{3}{*}{$\begin{array}{l}\text { blaDHA-1, } \\
\text { blaSHV-12 }\end{array}$} \\
\hline & ceftazidime & $0.5(\mathrm{~S})$ & & $0.25(\mathrm{~S})$ & & $0.5(\mathrm{~S})$ & & $>16(\mathrm{R})$ & \\
\hline & cefepime & $\leq 0.12(\mathrm{~S})$ & & $\leq 0.12(\mathrm{~S})$ & & $\leq 0.12(\mathrm{~S})$ & & $2(S)$ & \\
\hline monobactams & aztreonam & $\leq 0.12(\mathrm{~S})$ & & $\leq 0.12(\mathrm{~S})$ & & $\leq 0.12$ (S) & & $>16(\mathrm{R})$ & $\begin{array}{l}\text { blaDHA-1, } \\
\text { blaSHV-12 }\end{array}$ \\
\hline \multirow{2}{*}{ carbapenems } & meropenem & $\leq 0.12(\mathrm{~S})$ & & $\leq 0.12(S)$ & & $\leq 0.12(\mathrm{~S})$ & & $\leq 0.12(\mathrm{~S})$ & \\
\hline & ertapenem & $\leq 0.015(\mathrm{~S})$ & & $\leq 0.015$ (S) & & $\leq 0.015(\mathrm{~S})$ & & $0.03(\mathrm{~S})$ & \\
\hline \multirow{3}{*}{ aminoglycosides } & gentamicin & $0.5(\mathrm{~S})$ & & $0.5(\mathrm{~S})$ & \multirow{3}{*}{$\begin{array}{l}\operatorname{aph}\left(3^{\prime \prime}\right)-I b \\
\operatorname{aph}(6)-I d \\
\operatorname{aad} A 1\end{array}$} & $0.5(\mathrm{~S})$ & & $>32(\mathrm{R})$ & \multirow{3}{*}{$\begin{array}{l}\operatorname{aac}(3)-I I, \\
\operatorname{aac}\left(6^{\prime}\right)-I I c, \\
\operatorname{aph}(6)-I d, \\
\operatorname{aph}\left(3^{\prime \prime}\right)-I b\end{array}$} \\
\hline & amikacin & $\leq 1(\mathrm{~S})$ & & $2(S)$ & & $\leq 1(\mathrm{~S})$ & & $2(\mathrm{~S})$ & \\
\hline & tobramycin & $1(\mathrm{~S})$ & & $1(\mathrm{~S})$ & & $0.5(\mathrm{~S})$ & & $>8(\mathrm{R})$ & \\
\hline $\begin{array}{l}\text { trimethoprim- } \\
\text { sulfonamide }\end{array}$ & $\begin{array}{l}\text { trimethoprim- } \\
\text { sulfamethoxazole }\end{array}$ & $\begin{array}{l}0.06 / 1.19 \\
(\mathrm{~S})\end{array}$ & & $>4 / 76(\mathrm{R})$ & $\begin{array}{l}\text { sul1, sul2, } \\
\text { dfrA1 }\end{array}$ & $0.06 / 1.19(\mathrm{~S})$ & & $>4 / 76$ (R) & sul1, dfrA19 \\
\hline quinolones & ciprofloxacin & $\leq 0.06(\mathrm{~S})$ & & $\leq 0.06(\mathrm{~S})$ & & $\leq 0.06(\mathrm{~S})$ & & $0.25(\mathrm{R})$ & $q n r B 4$ \\
\hline amphenicols & chloramphenicol & $8(S)$ & & $4(\mathrm{~S})$ & & $>32(\mathrm{R})$ & cat $A 1$ & $4(\mathrm{~S})$ & \\
\hline tetracyclines & tetracycline & $2(S)$ & & $>32(\mathrm{R})$ & $\operatorname{tet}(A)$ & $>32(\mathrm{R})$ & $\operatorname{tet}(A)$ & $>32(\mathrm{R})$ & $\operatorname{tet}(B)$ \\
\hline
\end{tabular}

\subsection{Multilocus Sequence Typing and WGS-Typing}

The genomes of the sequenced isolates related to the ST and eBURST groups that correlated with the serotyping results, according to the analysis in EnteroBase using Achtman's seven gene MLST scheme [23]. The S. Enteritidis isolates SLR1_8250 and SLR1_8245 belonged to ST-11 and eBG-4; the S. Bovismorbificans isolate SLR1_7627 belonged to ST142 and eBG-34; and the monophasic variant of S. Typhimurium, isolate SLR1_8094, was related to ST-34 and eBG-1.

Although the seven gene MLST scheme [23] enables the determination of serovars of Salmonella isolates, it is not suitable for the determination of the phylogenetic positions of, and relatedness between, isolates. For such analyses, core genome analyses (SNP analyses) and core genome MLST are better suited. Thus, to further investigate the phylogenetic positions of our isolates in a global context, we used data publicity available in the NCBI Pathogen Detection system (https:/ / www.ncbi.nlm.nih.gov/pathogens/), which contains 398,518 genomes of Salmonella (accessed on 10 October 2021), as well as data in EnteroBase (https://enterobase.warwick.ac.uk/species/index/senterica), which contains 326,421 genomes of Salmonella (accessed on 10 October 2021). It should be noted that historical Russian isolates of $S$. Enteritids collected from outbreaks and sporadic cases during 2012-2020 were previously submitted to the NCBI Pathogen Detection system under bioproject PRJNA484865 (including SRL1_8239). Although the new Russian isolates investigated in the present study matched the ST of the seven gene MLST scheme (see above), the more detailed core genome analyses (SNP analyses and core genome MLST) did not reveal any close relationships to each other or to other isolates, except for the match between isolates SLR1_8245 and SLR1_8239 (which originated from the same outbreak); all other studied sporadic and outbreak isolates formed unique clades (orphans) differing by at least 50 SNP to other isolates in the NCBI Pathogen Detection system, or showed at least 10 allele differences by core genome MLST in EnteroBase. (Supplementary Figures S1-S4). 


\subsection{Related Genetic Determinants of Salmonella Isolates to Antimicrobial Susceptibility}

These four isolates were subjected to whole genome sequencing (WGS) using Illumina and Oxford Nanopore sequencing technology. Combining different WGS platforms provides the opportunity to reconstruct accurately all genomic elements, and to define the locations of plasmid-encoded genes responsible for colistin resistance, as well as additional sets of resistance genes (Table 3). Thus, we applied the PlasmidFinder and MOB-suite pipelines and the results are detailed below (Table 3).

Table 3. Genome content of completed genomes of Russian Salmonella isolates.

\begin{tabular}{|c|c|c|c|c|c|}
\hline $\begin{array}{l}\text { Serotype, Strain Name, } \\
\text { NCBI GenBank acc. }\end{array}$ & Replicon Name & Size, bp & AMR Genes ${ }^{1}$ & Replion Type(s) ${ }^{2}$ & $\begin{array}{l}\text { Mobility Prediction } \\
\text { for the Plasmid }^{3}\end{array}$ \\
\hline \multirow{4}{*}{$\begin{array}{l}\text { S. Enteritidis, SLR1_8250, } \\
\text { CP060522-CP060525 }\end{array}$} & chromosome & $4,679,617$ & ND & chromosome & ND \\
\hline & pS8250-1 & 59,372 & ND & $\operatorname{IncFII(S),~IncFIB(S)~}$ & non-mobilizable \\
\hline & pS8250-2 & 33,310 & $m c r-1.1$ & IncX4 & conjugative \\
\hline & pS8250-3 & 2096 & ND & ColpVC & mobilizable \\
\hline \multirow{4}{*}{ S. Enteritidis, SLR1_8245 } & chromosome & $4,680,323$ & ND & chromosome & - \\
\hline & pS8245-1 & 59,372 & ND & $\operatorname{IncFII(S),~IncFIB(S)~}$ & non-mobilizable \\
\hline & pS8245-2 & 56,813 & $\operatorname{cat} A 1, \operatorname{tet}(A)$ & IncX1 & conjugative \\
\hline & pS8245-3 & 33,310 & $m c r-1.1$ & IncX4 & conjugative \\
\hline \multirow{5}{*}{$\begin{array}{l}\text { S. Bovismorbificans, } \\
\text { SLR1_7627, } \\
\text { CP060517-CP060521 }\end{array}$} & chromosome & $4,715,485$ & ND & chromosome & ND \\
\hline & pS7627-1 & 233,305 & $\begin{array}{l}\text { aph(3")-Ib, aph }(6)-I d, \\
\text { aadA1, dfrA1, tet }(A), \\
\text { blaTEM-1B, sul1, sul2 }\end{array}$ & IncHI2, IncQ1 & non-mobilizable \\
\hline & pS7627-2 & 64,443 & $m c r-1.1$ & IncI2 & conjugative \\
\hline & pS7627-3 & 4073 & ND & rep_cluster_2350 ${ }^{3}$ & mobilizable \\
\hline & pS7627-4 & 3830 & ND & rep_cluster_2335 ${ }^{3}$ & mobilizable \\
\hline \multirow[b]{2}{*}{$\begin{array}{c}\text { S. Typhimurium [4,5:i:-], } \\
\text { SLR1_8094, } \\
\text { CP060515-CP060516 }\end{array}$} & chromosome & $5,017,156$ & $\operatorname{tet}(B)$ & chromosome & ND \\
\hline & pS8094-1 & 278,034 & $\begin{array}{c}\text { aph(3")-Ib, aph(6)-Id, } \\
\text { aac(3)-IIg, aac (6')-IIc, } \\
\operatorname{ere}(A), \text { qnrB4, } \\
\text { dfrA19,mcr-9, blaSHV-12, } \\
\text { blaDHA-1, sul1 x2 }\end{array}$ & IncHI2 & conjugative \\
\hline
\end{tabular}

ND—not detected; ${ }^{1}$ Predicted by ResFinder; ${ }^{2}$ Predicted by PlasmidFinder; ${ }^{3}$ Predicted by MOB-suite.

\subsubsection{Salmonella Enteritidis SLR1_8250 and SLR1_8245}

S. Enteritidis SLR1_8250 carried three plasmid types (Table 3), namely, a non-mobilizable $60 \mathrm{~kb}$ plasmid pS8250-1 of the IncFII(S)/IncFIB(S) type, a mobilizable small $2 \mathrm{~kb}$ plasmid pS8250-3 of the ColpVC type, and the conjugative plasmid pS8250-2 of IncX4 type (33 kb). The last one contains the phosphoethanolamine transferase gene ( $m c r-1.1$, red arrow in Figure 1), which was located upstream (4-5 kb) of a mobile genomic element of the IS26family. However, there was no copy of the ISApl1 insertion sequence flanking the $m c r-1.1$ gene. The sequence of a PAP2 family protein that is associated with mcr-1.1 was located directly downstream of $m c r-1.1$ (green arrow in Figure 1). This IncX4 plasmid also has a standard plasmid backbone containing sequences that are involved in plasmid replication, maintenance, and transfer, as well as genes responsible for the toxin-antitoxin system. 


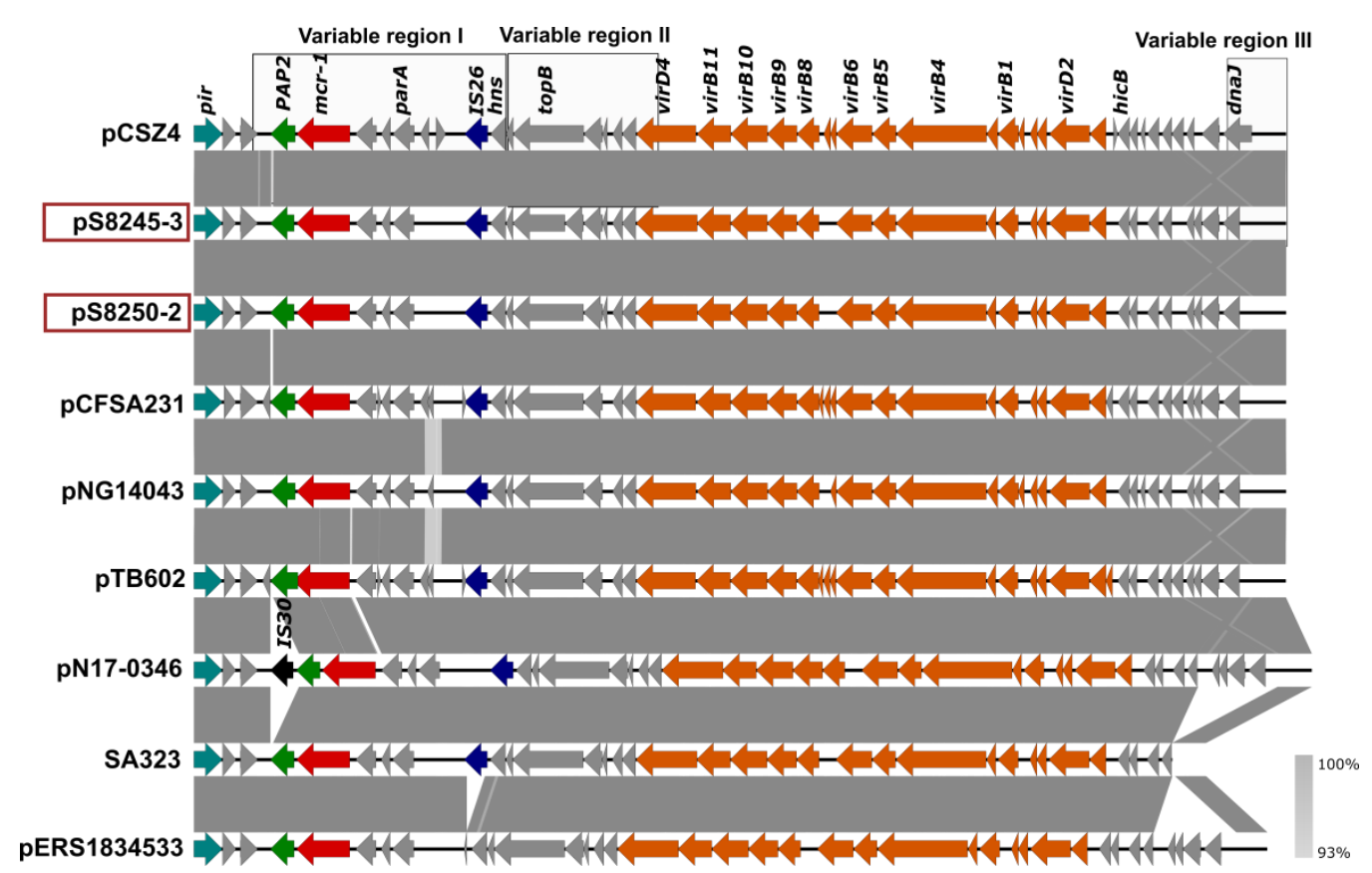

Figure 1. Comparison of the IncX4 plasmids from SLR1_8245, SLR1_8250, and Salmonella isolates with previously sequenced IncX4. GenBank accession numbers for these plasmids are listed in Supplementary Table S1. Arrows represent the position of open reading frames. Regions of $>99 \%$ identity are indicated in dark grey between adjacent plasmid sequences. Genes associated with the tra and pil loci are colored orange, replication associated genes are colored turquoise, antibiotic resistance genes are colored red, the PAP2 gene is colored green, insertion sequences are colored blue and black, and other genes are colored gray. The previously defined variable regions I, II, and III [30] were marked by square boxes.

The S. Enteritidis SLR1_8245 outbreak strain had three plasmids pS8245-1 (IncFII(S)/ IncFIB(S); 60kb), pS8245-2 (IncX1; 57kb), and pS8245-3 (IncX4; 33kb) (Table 3). The catA1 and $\operatorname{tet}(A)$ genes responsible for resistance to amphenicols and tetracyclines were located on the IncX1 plasmid. Plasmid pS8245-3 (IncX4) carried the mcr-1.1 and was similar with $100 \%$ coverage and $100 \%$ sequence identity to the IncX4 plasmid of isolate SLR1_8250 (Figure 1).

Compared to the non-redundant collection of sequences (NT) representing genomes in the NCBI GenBank database the complete IncX4 sequence was 99\% identical to and had a coverage from $92-100 \%$ of the IncX4 plasmids of E. coli (78 BLAST hits), K. pneumonia (12 BLAST hits), and S. enterica (11 BLAST hits), showing the prevalence of such a type of IncX4 plasmid among different species of Enterobacteriaceae. Comparative analysis with a set of previously sequenced IncX4 plasmids showed a high similarity level (100\% coverage and 99.98\% sequence identity) of pS8245-3 and pS8250-2 IncX4 plasmids to a so-called epidemic IncX4 plasmid pCSZ4 [30]. The similar IncX4 plasmids pCFSA231, pNG14043, and pTB602 have also been found in other NTS isolates [31,32] (Figure 1, Supplementary Table S1).

\subsubsection{Salmonella Bovismorbificans SLR1_7627}

The MDR colistin-resistant strain S. Bovismorbificans SLR1_7627 isolated from human samples in 2018 carried four plasmids of which only two carried resistance genes (Table 3). The IncI2 conjugative $64 \mathrm{~kb}$ plasmid (pS7627-2) included mcr-1.1, while additional resistance genes were located on the $233 \mathrm{~kb}$ non-mobilizable plasmid pS7627-1 of the IncHI2/IncQ1 type.

The results of the AST correlated well with identified genes responsible for resistance to antibiotics (Tables 2 and 3). Trimethoprim-sulfamethoxazole resistance is explained by the presence of $s u l 1$, sul2, and $d f r A 1$ genes. The resistance of the isolate to tetracycline is 
confirmed by presence of a tet $(A)$ gene. As well, the identified blaTEM-1B gene enables resistance to penicillins. However, the roles of genes aadA1, aph(6)-Id, and gene aph(3")-Ib, which encode proteins for streptomycin and spectinomycin resistance and resistance to kanamycin, respectively, could not be confirmed due to the absence of these antimicrobial agents in the panel.

A BLASTN comparison of the complete sequence of plasmid IncI2 to the NCBI GenBank database revealed similar plasmids in E. coli, S. Typhimurium, and K. aerogenes isolates with an identity of $99 \%$ and a query cover from $97-56 \%$. No further isolates of the serotype $S$. Bovismorbificans carrying similar plasmids were identified. Comparisons with previously published Salmonella and E. coli IncI2 plasmids (Supplementary Table S1), carrying mcr-1.1 gene, are shown in Figure 2. The IncI2 plasmid pS7627-2 has a similar plasmid backbone and a similar genetic environment surrounding the $m c r-1.1$ gene (nikBmor-1.1-PAP) compared to other IncI2 plasmids (pCESA664-3, pCESA244-2, pK18JST013) previously isolated in China and Korea [33]. The ISApl1 insertion sequence belonging to the IS30 family was not identified near mcr-1.1.

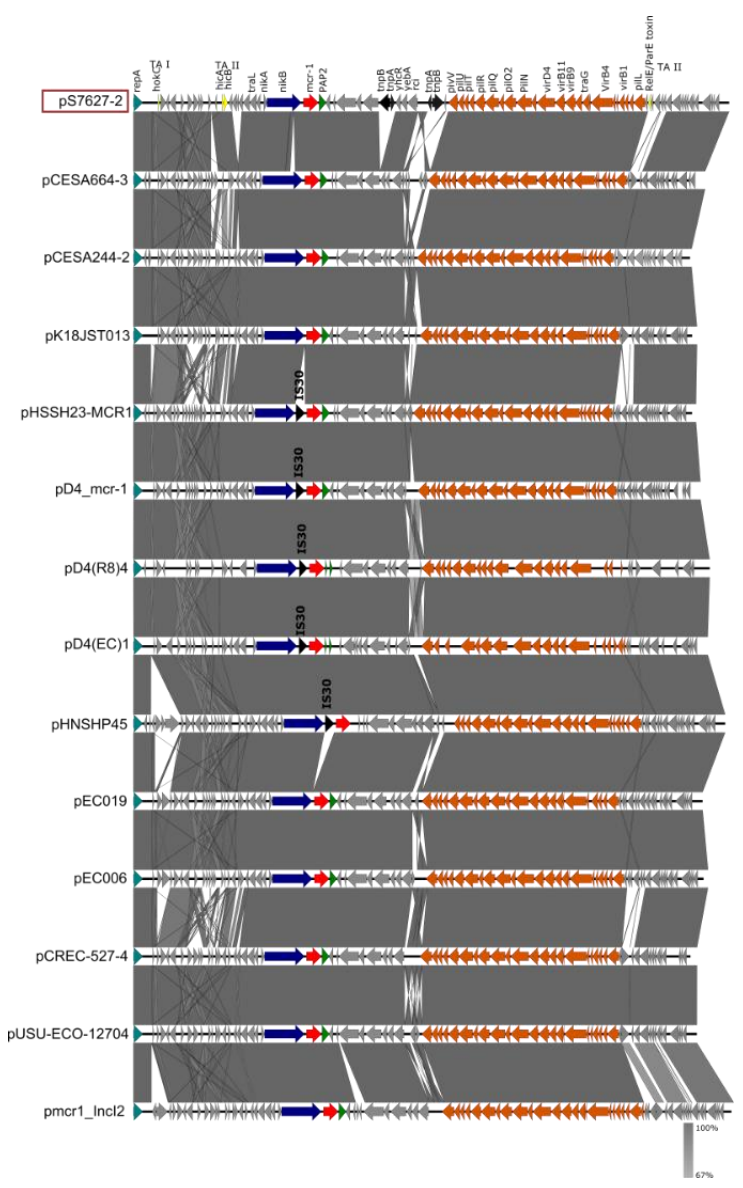

Figure 2. Comparison of the IncI2 plasmid of the pS7627-2 isolate carrying the $m c r-1.1$ gene to the IncI2 plasmids of Salmonella (pCESA664-3, pCESA244-2, pK18JST013, pHSSH23-MCR1, pD4_mcr-1, pD4(R8)4, pD4(EC)1) and E. coli (pHNSHP45, pEC019, pEC006, pCREC-527-4, pUSU-ECO-12704, pmcr1_IncI2) isolates. Arrows represent the position of open reading frames. The GenBank accession numbers for these plasmids are listed in Supplementary Table S1. Regions of $>99 \%$ identity are indicated in dark grey in the bar between compared plasmid sequences. The RepA gene is colored turquoise, genes associated with the plasmid backbone are colored orange, antibiotic resistance genes are colored red, the PAP2 gene is colored green, insertion sequences are colored black, genes encoding toxin-antitoxin (TA) systems of type I (TA-I) and type II (TA-II) are colored yellow, and other genes are colored gray. 


\subsubsection{Monophasic Variant of Salmonella Typhimurium 4,5:i:- (SLR1_8094)}

The MDR colistin-susceptible, ESBL-producing $S$. Typhimurium 4,5:i:- isolate SLR1_8094 had only the $278 \mathrm{~kb}$ conjugative plasmid pS8094-1 of the type IncHI2 (Table 3). Comparison of the full plasmid sequence of pS8094-1 against the NCBI GenBank database showed that it has a high similarity and query coverage to other IncHI2 plasmids from bacteria of nonSalmonella origin, e.g., E. hormaechei pHI2-233 (97\% coverage and 99\% sequence identity; CP049047.1); K. pneumoniae pCNR48 (97\% coverage and 99\% sequence identity; LT994835.1); C. sakazakii p505108-MDR (97\% coverage and 99\% sequence identity; KY978628 [34]); E. cloacae pIMP26 (95\% coverage and 99\% sequence identity; MH399264.1); and C. freundii pR47-309 (95\% coverage and 99\% sequence identity; CP040696.1 [35]). Similar plasmids in Salmonella had 99\% sequence identity but relatively low query coverage: pSA20094620.1 (83\% coverage; CP030186) and pXXB1403 (81\% coverage; CP059887.1). The plasmid pS80941 possesses the core backbone markers of typical IncHI2 plasmids, including genes encoding replication initiation, par $A B$ and parMR for partition, the tra1 and tra2 regions for conjugal transfer, and the tellurite resistance region (terY3Y $X Y Y 1 W Z A B C D E F)$ (Figure 3) [36].

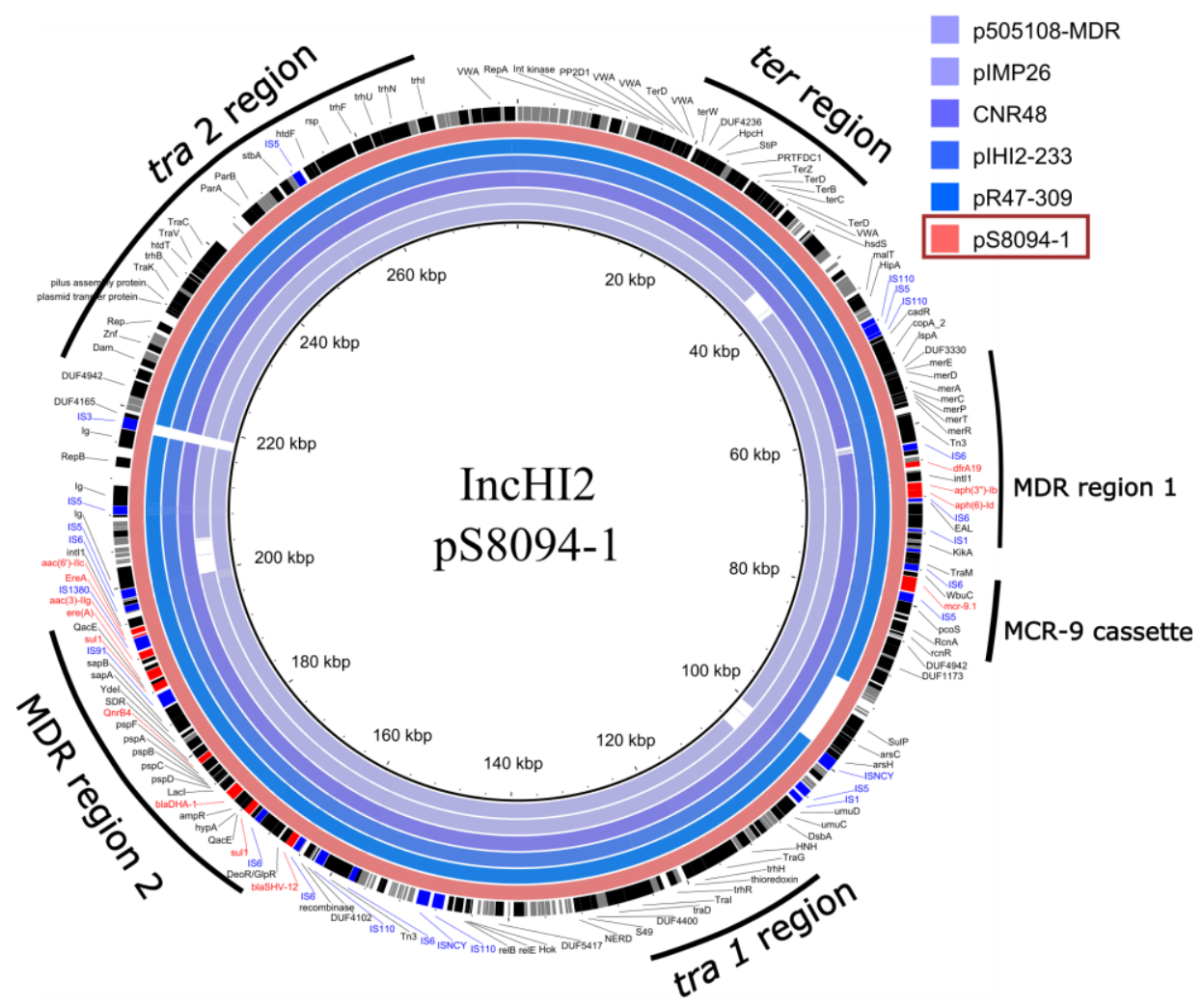

Figure 3. Circular map of the BLASTN comparison of IncHI2 type plasmids pS80904-1 (S. Typhimurium), pR47-309 (C. freundii), pHI2-233 (E. hormaechei), CNR48 (K. pneumoniae), pIMP26 (E. cloacae), and p505108-MDR (C. sakazakii) generated by the BLAST Ring Image Generator tool. The pS80904-1 plasmid was used as a reference. The outer circle denotes the annotation of the reference plasmid. The AMR genes are highlighted by red boxes, genes encoding hypothetical proteins highlighted by grey boxes, IS-elements highlighted by blue boxes, and other genes highlighted by black boxes. The backbone (tra1 and tra2 region), two accessory resistance regions (MDR region 1, MDR region 2), and $m c r-9$ cassette ( $r c n R-r c n A-p c o E-p c o S-I S 5-m c r-9-w b u C$-IS6) are indicated by black curves.

The phenotypic resistance to the different classes of antimicrobial agents, as well as the production of ESBL correlated with the identified AMR genes on the IncHI2 plasmid and chromosome (Table 2). The gene responsible for tetracycline resistance tet $(B)$ was located on the chromosome (Table 3). The IncHI2 plasmid carried genes responsible for resistance to aminoglycosides (aac( $\left.3^{\prime \prime}\right)$-IIg, aac(6')-IIc, aph(6)-Id, aph(3")-Ib), sulphonamide 
and trimethoprim (sul1 x2,dfrA19), quinolone (qnrB4), macrolides (ere $(A))$, and $\beta$-lactam antibiotics (blaDHA-1, blaSHV-12). These AMR genes were located in two different MDR regions (MDR region 1 and MDR region 2) on the IncHI2 plasmid (Figure 3). The identified $m c r$ cassette contained an mcr-9 gene located near the MDR region 1 . The genetic environment surrounding $m c r-9$ was $r c n R-r c n A-p c o E-p c o S-I S 5-m c r-9-w b u C$ with the IS6 element, which relates to the $m c r-9$ cassette type on plasmid p17277A_477 (IncHI2) according to the classification suggested by Li et al., 2020 [37]. This $m c r$ cassette lacks the downstream regulatory genes ( $q s e C$ and $q s e B$ ), which were proposed to be involved in the induction of the colistin resistance mediated by $m c r-9$ [37].

\section{Discussion}

The novelty of our study is the detection and description of four Russian NTS isolates at a genomic level, revealing details about plasmids carrying mor genes. Three isolates belong to $S$. Enteritidis $(n=2)$ and $S$. Bovismobificans $(n=1)$, and all of them were characterized by the presence of an $m c r-1.1$ gene and had phenotypical resistance to colistin. Colistin is one of the so-called "last-resort" antimicrobials used to treat MDR infections caused by members of the Enterobacteriaceae family. The fourth isolate belonged to monophasic $S$. Typhimurium and, while carrying an mcr-9 gene, was susceptible to colistin. The successful combination of the genome sequencing approaches based on short and long reads allowed us to accurately reconstruct the complete sequences of the chromosomes and plasmids for each isolate, permitting detailed analyses of the plasmid's structure that carries the $\mathrm{mcr}$ genes. To the best of our knowledge, this is the first study that describes cases of NTS strain isolation with mcr-mediated resistance to colistin in Russia with a detailed analysis of both AMR genes and phenotypical resistances.

Based on the data obtained, we conclude that the prevalence rate of mcr-1.1 in Salmonella isolates is very low $(0.5 \%)$, which correlates well with previous observations [38,39]. Nevertheless, three of the studied isolates were phenotypically resistant to at least three classes of antimicrobial drugs. These MDR phenotypes were accurately confirmed by the presence of corresponding AMR genes in the genomes, corroborating the predictive power of WGS [40]. Based on the WGS data, we show that the sequenced isolates are genetically unique by core genome features (SNP and alleles of core genes) among all available genomes sequenced to date for Salmonella. Moreover, we did not find any similarity to previously isolated $S$. Enteritidis representatives from the outbreaks and sporadic cases circulating in Russia.

Based on the analyzed genomic sequences, we found that the $m c r-1.1$ genes of two $S$. Enteridis isolates were localized on IncX4 plasmids, whilst in the $S$. Bovismorbificans isolate the $m c r-1.1$ was on the IncI2 plasmid. An $m c r-9$ gene variant was detected on the IncHI2 plasmid in the monophasic human isolate of $S$. Typhimurium. The data obtained are consistent with previous observations, where it was shown that plasmid types predominantly harboring mor genes are IncX4, IncI2, and IncHI2 [39,41,42]. Interestingly, only the IncHI2 plasmid was associated with additional antibiotic resistance genes, while the IncX 4 and IncI2 plasmids carried only one $\mathrm{mcr}$ gene. The absence of a flanking ISApl1 near the $m c r$ cassettes on the IncX4 and IncI2 plasmids suggest loss of the ISApl1 elements after the integration of the transposon. It further suggests that the loss of ISApl1 anchors the $m c r-1$ gene on the plasmid, which probably indicates the stability of the $m c r$ genes on plasmids [6].

By comparing the plasmid sequences with previously published data, we found that the IncX4 plasmids in the two $S$. Enteritidis strains in this study showed high levels of similarity to an epidemic pCSZ4-like IncX4 plasmid [30]. The IncX4 epidemic plasmid pCSZ4 contains an identical mor cassette with absence of the ISApl1 transposon. It should be noted that IncX4 is one of the most widespread plasmid types among E. coli and other Enterobacteriaceae. This plasmid is characterized by a high frequency of self-transfer between different species of Enterobacteriaceae, indicating its high epidemiological significance [43]. It was also interesting to note that $m c r-1.1$ positive isolates were found among the Salmonella isolates belonging to the serotype Enteritidis, which is the dominant etiological serotype for 
morbidity worldwide $[44,45]$. In the present study, $S$. Enteritidis were isolated during an outbreak of salmonellosis and from a poultry product, which indicates the opportunity for successful circulation of epidemic pCSZ4-like IncX4 plasmids in S. Enteritidis populations. To date, there are only a few cases of $S$. Enteritidis described with plasmids carrying $m c r$ genes, but none of these studies detected the epidemic IncX4 plasmid in $S$. Enteritidis isolates $[38,46]$.

Remarkably, the isolate carrying the $m c r-9$ gene (monophasic S.Typhimurium) did not show phenotypical resistance to colistin. According to earlier studies $[47,48]$, this phenomenon was previously observed, although colistin-resistant Salmonella enterica carrying the $m c r-9$ gene was also discovered [49]. Obviously, $m c r-9$ is widely distributed among various Enterobacteriaceae species, and the most common $m c r-9$ carrying plasmid is shown to be IncHI2 [37]. Moreover, co-occurrence of the $m c r-9$ gene with various antibiotic resistance genes was shown, indicating the epidemiological significance of IncHI2 plasmids in spreading of AMR genes [50]. Most often, and consistent with our data, the structure of the mcr-9 cassettes in Salmonella plasmids presents as IS5-mcr-9-wbuC-IS6 and lacks the downstream regulatory genes $q s e B$ and $q s e C$ [48,50].

The data provided here expand the global picture fordissemination of mobilized colistin resistance in Enterobacteriaceae. However, future retrospective studies from different sources over a longer period of time are urgently needed to assess the prevalence of plasmid-mediated colistin resistance among NTS strains in Russia.

Supplementary Materials: The following are available online at https://www.mdpi.com/article/10.3 390/microorganisms9122515/s1. Table S1: Detailed information of IncX4, IncI2 plasmids used for comparative analysis. Figures S1-S4: Defining the uniqueness of isolates using the NCBI Pathogen Detection system and EnteroBase.

Author Contributions: Conceptualization, K.V.K. and A.T.P.; Methodology, K.V.K., A.S.P., Y.V.M., E.V.K.; Validation, K.V.K., A.S.P.; Formal Analysis, K.V.K., G.M., S.H., I.V.C.; Data Curation, K.V.K.; Writing-Original Draft Preparation, K.V.K., E.D.S., G.M., S.H. Editing, K.V.K., G.M., S.H., E.D.S., V.G.A. Supervision, A.T.P., V.G.A. All authors have read and agreed to the published version of the manuscript.

Funding: This research received no external funding.

Institutional Review Board Statement: Ethical review and approval were waived for this study since the human samples were routinely collected and patients' data remained anonymous.

Informed Consent Statement: Patient consent was waived since the human samples were routinely collected, and patients' data remained anonymous.

Data Availability Statement: Raw sequencing reads of strains as well as complete genome assemblies have been deposited in the NCBI BioProject database under accession numbers PRJNA484865 $($ SAMN15666299 = SLR1_8250, SAMN15666300 = SLR1_7627, SAMN15666301 = SLR1_8094, SAMN20345806 = SLR1_8245, SAMN20345807 = SLR1_8239).

Conflicts of Interest: The authors declare no conflict of interest.

\section{References}

1. Centers for Disease Control and Prevention. National Salmonella Surveillance Annual Report, 2011. Atlanta, Georgia: US Department of Health and Human Services, CDC. 2013. Available online: https://www.cdc.gov/ncezid/dfwed/PDFs/salmonellaannual-report-2011-508c.pdf (accessed on 3 December 2021).

2. Majowicz, S.E.; Musto, J.; Scallan, E.; Angulo, F.J.; Kirk, M.; O’Brien, S.J.; Jones, T.F.; Fazil, A.; Hoekstra, R.M.; International Collaboration on Enteric Disease “Burden of Illness" Studies. The global burden of nontyphoidal Salmonella gastroenteritis. Clin. Infect. Dis. 2010, 50, 882-889. [CrossRef]

3. State Report on the State of Sanitary and Epidemiological Well-Being of the Population in the Russian Federation in 2020. Available online: https:/ / www.rospotrebnadzor.ru/bitrix/redirect.php?event1=file\&event2=download\&event3=gd-seb_02.0 6-_s-podpisyu_.pdf\&goto=/upload/iblock/5fa/gd-seb_02.06-_s-podpisyu_.pdf (accessed on 1 November 2021). (In Russian).

4. Rozhnova, S.S.; Kuleshov, K.V.; Pavlova, A.S.; Guseva, A.N.; Kozhakhmetova, T.A.; Akulova, N.K.; Podkolzin, A.T. Heterogeneity of Salmonella isolates obtained from various sources in Russian Federation 2010-2019. Epidemiol. Infect. Dis. 2020, 25, 26-34. (In Russian) [CrossRef] 
5. Caniaux, I.; van Belkum, A.; Zambardi, G.; Poirel, L.; Gros, M.F. MCR: Modern colistin resistance. Eur. J. Clin. Microbiol. Infect. Dis. 2017, 36, 415-420. [CrossRef] [PubMed]

6. Wang, R.; van Dorp, L.; Shaw, L.P.; Bradley, P.; Wang, Q.; Wang, X.; Jin, L.; Zhang, Q.; Liu, Y.; Rieux, A.; et al. The global distribution and spread of the mobilized colistin resistance gene mcr-1. Nat. Commun. 2018, 9, 1179. [CrossRef]

7. Lima, T.; Domingues, S.; Da Silva, G.J. Plasmid-Mediated Colistin Resistance in Salmonella enterica: A Review. Microorganisms 2019, 7, 55. [CrossRef]

8. Scott, H.M.; Acuff, G.; Bergeron, G.; Bourassa, M.W.; Gill, J.; Graham, D.W.; Kahn, L.H.; Morley, P.S.; Salois, M.J.; Simjee, S.; et al. Critically important antibiotics: Criteria and approaches for measuring and reducing their use in food animal agriculture. Ann. N. Y. Acad. Sci. 2019, 1441, 8-16. [CrossRef]

9. Liu, Y.Y.; Wang, Y.; Walsh, T.R.; Yi, L.X.; Zhang, R.; Spencer, J.; Doi, Y.; Tian, G.B.; Dong, B.L.; Huang, X.H.; et al. Emergence of plasmid-mediated colistin resistance mechanism MCR-1 in animals and human beings in China: A microbiological and molecular biological study. Lancet Infect. Dis. 2016, 16, 161-168. [CrossRef]

10. Poirel, L.; Jayol, A.; Nordmann, P. Polymyxins: Antibacterial Activity, Susceptibility Testing, and Resistance Mechanisms Encoded by Plasmids or Chromosomes. Clin. Microbiol. Rev. 2017, 30, 557-596. [CrossRef]

11. Vallejos-Sanchez, K.; Tataje-Lavanda, L.; Villanueva-Perez, D.; Bendezu, J.; Montalvan, A.; Zimic-Peralta, M.; Fernandez-Sanchez, M.; Fernandez-Diaz, M. Whole-Genome Sequencing of a Salmonella enterica subsp. enterica Serovar Infantis Strain Isolated from Broiler Chicken in Peru. Microbiol. Resour. Announc. 2019, 8, e00826-19. [CrossRef] [PubMed]

12. Rozwandowicz, M.; Brouwer, M.S.M.; Fischer, J.; Wagenaar, J.A.; Gonzalez-Zorn, B.; Guerra, B.; Mevius, D.J.; Hordijk, J. Plasmids carrying antimicrobial resistance genes in Enterobacteriaceae. J. Antimicrob. Chemother. 2018, 73, 1121-1137. [CrossRef] [PubMed]

13. Datta, N.; Hughes, V.M. Plasmids of the same Inc groups in Enterobacteria before and after the medical use of antibiotics. Nature 1983, 306, 616-617. [CrossRef] [PubMed]

14. Matamoros, S.; van Hattem, J.M.; Arcilla, M.S.; Willemse, N.; Melles, D.C.; Penders, J.; Vinh, T.N.; Hoa, N.T.; Bootsma, M.C.J.; van Genderen, P.J.; et al. Global phylogenetic analysis of Escherichia coli and plasmids carrying the mcr-1 gene indicates bacterial diversity but plasmid restriction. Sci. Rep. 2017, 7, 15364. [CrossRef]

15. Doumith, M.; Godbole, G.; Ashton, P.; Larkin, L.; Dallman, T.; Day, M.; Day, M.; Muller-Pebody, B.; Ellington, M.J.; de Pinna, E.; et al. Detection of the plasmid-mediated mcr-1 gene conferring colistin resistance in human and food isolates of Salmonella enterica and Escherichia coli in England and Wales. J. Antimicrob. Chemother. 2016, 71, 2300-2305. [CrossRef] [PubMed]

16. Manageiro, V.; Clemente, L.; Romao, R.; Silva, C.; Vieira, L.; Ferreira, E.; Canica, M. IncX4 Plasmid Carrying the New mcr-1.9 Gene Variant in a CTX-M-8-Producing Escherichia coli Isolate Recovered from Swine. Front. Microbiol. 2019, 10, 367. [CrossRef]

17. Ye, H.; Li, Y.; Li, Z.; Gao, R.; Zhang, H.; Wen, R.; Gao, G.F.; Hu, Q.; Feng, Y. Diversified mcr-1-Harbouring Plasmid Reservoirs Confer Resistance to Colistin in Human Gut Microbiota. MBio 2016, 7, e00177. [CrossRef] [PubMed]

18. European Committee on Antimicrobial Susceptibility Testing. Breakpoint Tables for Interpretation of MICs and Zone Di-ameters. Version 11.0, valid from 1 January 2021. Available online: https://www.eucast.org/fileadmin/src/media/PDFs/EUCAST_files/ Breakpoint_tables/v_11.0_Breakpoint_Tables.pdf (accessed on 29 November 2021).

19. EUCAST. Guidelines for Detection of Resistance Mechanisms and Specific Resistances of Clinical and/or Epidemiological Importance. Version 2.0. Available online: https://www.eucast.org/fileadmin/src/media/PDFs/EUCAST_files/Resistance_ mechanisms/EUCAST_detection_of_resistance_mechanisms_170711.pdf (accessed on 29 November 2021).

20. Wick, R.R.; Judd, L.M.; Gorrie, C.L.; Holt, K.E. Unicycler: Resolving bacterial genome assemblies from short and long sequencing reads. PLoS Comput. Biol. 2017, 13, e1005595. [CrossRef] [PubMed]

21. Koren, S.; Walenz, B.P.; Berlin, K.; Miller, J.R.; Bergman, N.H.; Phillippy, A.M. Canu: Scalable and accurate long-read assembly via adaptive k-mer weighting and repeat separation. Genome Res. 2017, 27, 722-736. [CrossRef] [PubMed]

22. Seibt, K.M.; Schmidt, T.; Heitkam, T. FlexiDot: Highly customizable, ambiguity-aware dotplots for visual sequence analyses. Bioinformatics 2018, 34, 3575-3577. [CrossRef]

23. Achtman, M.; Wain, J.; Weill, F.X.; Nair, S.; Zhou, Z.M.; Sangal, V.; Krauland, M.G.; Hale, J.L.; Harbottle, H.; Uesbeck, A.; et al. Multilocus Sequence Typing as a Replacement for Serotyping in Salmonella enterica. PLoS Pathog. 2012, 8, e1002776. [CrossRef]

24. Robertson, J.; Nash, J.H.E. MOB-suite: Software tools for clustering, reconstruction and typing of plasmids from draft assemblies. Microb. Genom. 2018, 4, e000206. [CrossRef]

25. Carattoli, A.; Zankari, E.; Garcia-Fernandez, A.; Larsen, M.V.; Lund, O.; Villa, L.; Aarestrup, F.M.; Hasman, H. In Silico Detection and Typing of Plasmids Using PlasmidFinder and Plasmid Multilocus Sequence Typing. Antimicrob. Agents Chemother. 2014, 58, 3895-3903. [CrossRef] [PubMed]

26. Bortolaia, V.; Kaas, R.S.; Ruppe, E.; Roberts, M.C.; Schwarz, S.; Cattoir, V.; Philippon, A.; Allesoe, R.L.; Rebelo, A.R.; Florensa, A.F.; et al. ResFinder 4.0 for predictions of phenotypes from genotypes. J. Antimicrob. Chemother. 2020, 75, 3491-3500. [CrossRef] [PubMed]

27. Feldgarden, M.; Brover, V.; Gonzalez-Escalona, N.; Frye, J.G.; Haendiges, J.; Haft, D.H.; Hoffmann, M.; Pettengill, J.B.; Prasad, A.B.; Tillman, G.E.; et al. AMRFinderPlus and the Reference Gene Catalog facilitate examination of the genomic links among antimicrobial resistance, stress response, and virulence. Sci. Rep. 2021, 11, 12728. [CrossRef] [PubMed]

28. Sullivan, M.J.; Petty, N.K.; Beatson, S.A. Easyfig: A genome comparison visualizer. Bioinformatics 2011, 27, 1009-1010. [CrossRef] [PubMed] 
29. Alikhan, N.F.; Petty, N.K.; Zakour, N.L.B.; Beatson, S.A. BLAST Ring Image Generator (BRIG): Simple prokaryote genome comparisons. BMC Genom. 2011, 12, 402. [CrossRef] [PubMed]

30. Sun, J.; Fang, L.X.; Wu, Z.; Deng, H.; Yang, R.S.; Li, X.P.; Li, S.M.; Liao, X.P.; Feng, Y.; Liu, Y.H. Genetic Analysis of the IncX4 Plasmids: Implications for a Unique Pattern in the mcr-1 Acquisition. Sci. Rep. 2017, 7, 424. [CrossRef]

31. Hu, Y.; Nguyen, S.V.; Wang, W.; Gan, X.; Dong, Y.; Liu, C.; Cui, X.; Xu, J.; Li, F.; Fanning, S. Antimicrobial Resistance and Genomic Characterization of Two mcr-1-Harboring Foodborne Salmonella Isolates Recovered in China, 2016. Front. Microbiol. 2021, 12, 636284. [CrossRef]

32. Tang, B.; Chang, J.; Zhang, L.; Liu, L.; Xia, X.; Hassan, B.H.; Jia, X.; Yang, H.; Feng, Y. Carriage of Distinct mcr-1-Harboring Plasmids by Unusual Serotypes of Salmonella. Adv. Biosyst. 2020, 4, e1900219. [CrossRef]

33. Moon, D.C.; Kim, S.J.; Mechesso, A.F.; Kang, H.Y.; Song, H.J.; Choi, J.H.; Yoon, S.S.; Lim, S.K. Mobile Colistin Resistance Gene mcr-1 Detected on an IncI2 Plasmid in Salmonella Typhimurium Sequence Type 19 from a Healthy Pig in South Korea. Microorganisms 2021, 9, 398. [CrossRef]

34. Shi, L.; Liang, Q.; Zhan, Z.; Feng, J.; Zhao, Y.; Chen, Y.; Huang, M.; Tong, Y.; Wu, W.; Chen, W.; et al. Co-occurrence of 3 different resistance plasmids in a multi-drug resistant Cronobacter sakazakii isolate causing neonatal infections. Virulence 2018, 9, 110-120. [CrossRef]

35. Zhou, W.; Chen, Q.; Qian, C.; Shen, K.; Zhu, X.; Zhou, D.; Lu, W.; Sun, Z.; Liu, H.; Li, K.; et al. In Vitro Susceptibility and Florfenicol Resistance in Citrobacter Isolates and Whole-Genome Analysis of Multidrug-Resistant Citrobacter freundii. Int. J. Genom. 2019, 2019, 7191935. [CrossRef]

36. Johnson, T.J.; Wannemeuhler, Y.M.; Scaccianoce, J.A.; Johnson, S.J.; Nolan, L.K. Complete DNA sequence, comparative genomics, and prevalence of an IncHI2 plasmid occurring among extraintestinal pathogenic Escherichia coli isolates. Antimicrob. Agents Chemother. 2006, 50, 3929-3933. [CrossRef] [PubMed]

37. Li, Y.; Dai, X.; Zeng, J.; Gao, Y.; Zhang, Z.; Zhang, L. Characterization of the global distribution and diversified plasmid reservoirs of the colistin resistance gene mcr-9. Sci. Rep. 2020, 10, 8113. [CrossRef] [PubMed]

38. Cui, M.; Zhang, J.; Gu, Z.; Li, R.; Chan, E.W.; Yan, M.; Wu, C.; Xu, X.; Chen, S. Prevalence and Molecular Characterization of mcr-1-Positive Salmonella Strains Recovered from Clinical Specimens in China. Antimicrob. Agents Chemother. 2017, 61, e02471-16. [CrossRef] [PubMed]

39. Sia, C.M.; Greig, D.R.; Day, M.; Hartman, H.; Painset, A.; Doumith, M.; Meunier, D.; Jenkins, C.; Chattaway, M.A.; Hopkins, K.L.; et al. The characterization of mobile colistin resistance ( $\mathrm{mcr}$ ) genes among 33,000 Salmonella enterica genomes from routine public health surveillance in England. Microb. Genom. 2020, 6, e000331. [CrossRef]

40. Cooper, A.L.; Low, A.J.; Koziol, A.G.; Thomas, M.C.; Leclair, D.; Tamber, S.; Wong, A.; Blais, B.W.; Carrillo, C.D. Systematic Evaluation of Whole Genome Sequence-Based Predictions of Salmonella Serotype and Antimicrobial Resistance. Front. Microbiol. 2020, 11, 549. [CrossRef]

41. Migura-Garcia, L.; Gonzalez-Lopez, J.J.; Martinez-Urtaza, J.; Sanchez, J.R.A.; Moreno-Mingorance, A.; Rozas, A.P.d.; Hofle, U.; Ramiro, Y.; Gonzalez-Escalona, N. mcr-Colistin Resistance Genes Mobilized by IncX4, IncHI2, and IncI2 Plasmids in Escherichia coli of Pigs and White Stork in Spain. Front. Microbiol. 2019, 10, 3072. [CrossRef] [PubMed]

42. Wu, R.; Yi, L.X.; Yu, L.F.; Wang, J.; Liu, Y.; Chen, X.; Lv, L.; Yang, J.; Liu, J.H. Fitness Advantage of mcr-1-Bearing IncI2 and IncX4 Plasmids In Vitro. Front. Microbiol. 2018, 9, 331. [CrossRef]

43. Lo, W.U.; Chow, K.H.; Law, P.Y.; Ng, K.Y.; Cheung, Y.Y.; Lai, E.L.; Ho, P.L. Highly conjugative IncX4 plasmids carrying blaCTX-M in Escherichia coli from humans and food animals. J. Med. Microbiol. 2014, 63, 835-840. [CrossRef]

44. Rodrigue, D.C.; Tauxe, R.V.; Rowe, B. International increase in Salmonella enteritidis: A new pandemic? Epidemiol. Infect. 1990, 105, 21-27. [CrossRef]

45. Christenson, J.C. Salmonella infections. Pediatr. Rev. 2013, 34, 375-383. [CrossRef] [PubMed]

46. Lu, X.; Zeng, M.; Xu, J.; Zhou, H.; Gu, B.; Li, Z.; Jin, H.; Wang, X.; Zhang, W.; Hu, Y.; et al. Epidemiologic and genomic insights on mcr-1-harbouring Salmonella from diarrhoeal outpatients in Shanghai, China, 2006-2016. EBioMedicine 2019, 42, 133-144. [CrossRef] [PubMed]

47. Carroll, L.M.; Gaballa, A.; Guldimann, C.; Sullivan, G.; Henderson, L.O.; Wiedmann, M. Identification of Novel Mobilized Colistin Resistance Gene mcr-9 in a Multidrug-Resistant, Colistin-Susceptible Salmonella enterica Serotype Typhimurium Isolate. MBio 2019, 10, e00853-19. [CrossRef]

48. Tyson, G.H.; Li, C.; Hsu, C.H.; Ayers, S.; Borenstein, S.; Mukherjee, S.; Tran, T.T.; McDermott, P.F.; Zhao, S. The mcr-9 Gene of Salmonella and Escherichia coli Is Not Associated with Colistin Resistance in the United States. Antimicrob. Agents Chemother. 2020, 64, e00573-20. [CrossRef]

49. Cha, M.H.; Woo, G.J.; Lee, W.; Kim, S.H.; Woo, J.H.; Kim, J.; Ryu, J.G.; Kwak, H.S.; Chi, Y.M. Emergence of Transferable mcr-9 Gene-Carrying Colistin-Resistant Salmonella enterica Dessau ST14 Isolated from Retail Chicken Meat in Korea. Foodborne Pathog. Dis. 2020, 17, 720-727. [CrossRef]

50. Wang, Y.; Li, Z.; Lyu, N.; Ma, S.; Liu, F.; Hu, Y.; Gao, G.F.; Zhu, B. Comparative genomic analysis of mobile colistin resistance gene mcr-9 in Salmonella enterica. J. Infect. 2021, 82, e15-e17. [CrossRef] [PubMed] 